\begin{tabular}{|c|l|}
\hline Title & $\begin{array}{l}\text { Coupling Planet Simulator Mars, a general circulation model of the Martian atmosphere, to the ice sheet model } \\
\text { SICOPOLIS }\end{array}$ \\
\hline Author(s) & Stenzel, Oliver J.; Grieger, Björn; Keller, Horst-Uwe; Greve, Ralf; Fraedrich, Klaus; Kirk, Edil lbert; Lunkeit, Frank \\
\hline Citation & $\begin{array}{l}\text { Planetary and Space Science, 55(14), 2087-2096 } \\
\text { https://doi.org/10.1016/.pss.2007.09.001 }\end{array}$ \\
\hline Issue Date & 2007-11 \\
\hline Doc URL & http://hdl.handle.net/2115/30176 \\
\hline Type & article (author version) \\
\hline File Information & PSS55-14.pdf \\
\hline
\end{tabular}

Instructions for use 


\title{
Coupling Planet Simulator Mars, a general circulation model of the Martian atmosphere, to the ice sheet model SICOPOLIS
}

\author{
Oliver J. Stenzel (1), Björn Grieger (1), \\ Horst-Uwe Keller (1), Ralf Greve (2), Klaus Fraedrich (3), \\ Edilbert Kirk (3) and Frank Lunkeit (3)
}

(1) Max Planck Institute for Solar System Research, Max-Planck-Straße 2, D-37191 Katlenburg-Lindau, Germany

(2) Institute of Low Temperature Science, Hokkaido University,

Kita-19, Nishi-8, Kita-ku, Sapporo 060-0819, Japan

(3) Meteorological Institute, University of Hamburg, Bundesstraße 55, D-20146 Hamburg, Germany

November 21, 2007

Correspondence to: O. J. Stenzel (stenzel@mps.mpg.de) 


\begin{abstract}
A general circulation model of the Martian atmosphere is coupled with a 3-dimensional polythermal ice-sheet model of the polar ice caps. With this combination a series of experiments is carried out to investigate the impact of long term obliquity change on the Martian north polar ice cap (NPC). The behaviour of the NPC is tested under obliquities of $\theta=15^{\circ}, 25^{\circ}$ and $35^{\circ}$. With increasing obliquity the area covered by the NPC gets smaller but does not vanish. However, when started from an ice free condition the models develop an ice cap only for low obliquities. The "critical" obliquity at which a build up of a new polar cap is possible is $\theta=22^{\circ}$.
\end{abstract}

\title{
1 Introduction
}

Like the Earth, Mars has polar ice caps. From satellite observation we know that the Martian polar regions show structures, the so called polar layer deposits, which lead to the assumption of periodic climate changes recorded within the polar caps. To understand how the layered deposits and the ice-caps evolve during Mars' strong orbit driven climate variations, numerical simulation of the involved physical systems atmosphere and ice are necessary. The residual ice caps and the underlying layered deposits are considered to be geomorphological units and shall be referred to as the north- and south-polar cap (NPC/SPC), respectively.

Established modelling groups work on Martian atmosphere at Laboratoire de Météorologie Dynamique du C.N.R.S. (LMD/CNRS) and the University of Oxford, Atmospheric, Oceanic and Planetary Physics (AOPP) on the European GCM (Read et al. 1997, Forget et al. 1999), at Ames Research Center on the NASA Ames Research centre's GCM (Pollack et al. 1990), and on the 'SKYHI' model (Richardson and Wilson 2002b). The models, all derivatives of Earth General Circulation Models (GCMs), solve the equations of fluid dynamics and include e.g. the processes of radiative transfer, cloud formation, regolith-atmosphere water exchange, and advective transport of dust and trace gases. They take into account the topography of the Martian surface and include the altitude region between 0 and about $80 \mathrm{~km}$ to $120 \mathrm{~km}$, depending on the specific model. These state-of-the-art models reproduce the observed temperature structure, pressure variations and water cycle reasonably well. Such models, however, have in common that they are designed to simulate the present atmosphere in as much detail as possible. Consequently, they are computationally too expensive to permit long term paleoclimate studies. The longest integrations so far, used to create the Martian Climate Database (Read et al. 1997), cover a few Martian years whereas paleostudies 
require integrations over a few million years to cover the Martian obliquity cycles with periods of $125 \mathrm{ky}$ and $1.3 \mathrm{My}$.

On the other side, simple one-dimensional models have been used to study specific processes that do not require full solution of the dynamic equations. Examples are the radiative transfer model by Gierasch and Goody (1968), the energy balance model by Armstrong et al. (2004), regolith-atmosphere water exchange by Jakosky (1985) and water ice formation by Michelangeli et al. (1993). Our model, the Planet Simulator Mars, is designed to close the gap between these simple models and the fully complex GCMs. Earth Models of Intermediate Complexity as well as simplified GCMs have successfully been used to simulate terrestrial climate transitions (e.g., Petoukhov et al. 2000, Stenzel and von Storch 2004).

A recalculation of the orbital parameters obliquity, eccentricity, and longitude of perihelion relative to the ascending node for the last 10 million years has been published recently by Laskar et al. $(2002,2004)$. These orbital parameters will be used to force our model in further experiments.

To understand the behaviour of the Martian ice caps at different climate conditions a second model is needed to simulate the ice physics. SICOPOLIS (SImulation COde for POLythermal Ice Sheets), a three-dimensional, dynamic/thermodynamic ice-sheet model, provides this capability, as can been seen in the studies Greve et al. (2004), Greve and Mahajan (2005) and Greve (2006). This paper presents the coupling of the atmospheric model Planet Simulator Mars with the polythermal ice-sheet model SICOPOLIS and some first results obtained with the coupled version. In section 2 and 3 , respectively, we describe the models and the experiments. In section 4 we present and discuss the results. Our conclusions are summarised in section 5.

\section{Model description and coupling}

\subsection{The models Planet Simulator Mars and SICOPOLIS}

The Planet Simulator is a circulation model, which includes the dynamical core, parameterisations and subsystems, and a graphical user interface with user friendly model starter and on the fly display and interaction. The complete model package including sources and documentation is available at www.mi.uni-hamburg.de/plasim. The Planet Simulator Earth (Fraedrich et al. 2005a), in particular its atmospheric module PUMA II, is the basis for the development of Planet Simulator Mars (Segschneider et al. 2005), which is a spectral circulation model of the Martian atmosphere. It simulates the atmospheric motions by integration of the primitive equations. Sub-scale processes are 
parameterised in simple numeric schemes. The ice-sheet model SICOPOLIS was developed in the mid-1990's for terrestrial applications (Greve 1997) and has later been adapted to the polar caps of Mars. It solves the prognostic ice-flow equations based on the shallow-ice approximation (Hutter 1983, Morland 1984). Inputs from the environment are specified by the mean annual surface temperature, the net surface mass balance (ice accumulation minus ablation, which can be equated to precipitation minus evaporation under Martian conditions) and the basal ("geothermal") heat flux from the underlying lithosphere. The numerical solution of the model equations is carried out by a finite-difference integration technique. A large part of the properties of the models Planet Simulator Mars and SICOPOLIS is described in Segschneider et al. (2005) and Greve et al. (2004), Greve and Mahajan (2005) and Greve (2006). Therefore this paper discusses only the differences made since the publication of the aforementioned papers. These changes constitute mainly of the coupling of the ice and atmosphere components and are covered extensively. Unlike the version described in Segschneider et al. (2005), the current version of Planet Simulator Mars now includes a hydrological cycle. The parametrisation is mostly identical to that of the original Planet Simulator Earth (Fraedrich et al. 2005b,a, Lunkeit et al. 2004). The water cycle is described below:

Water vapour is distributed within the atmosphere by advection, calculated from the corresponding prognostic equation and through vertical diffusion. The atmosphere loses water by condensation which takes place when the specific humidity reaches saturation. In Planet Simulator Earth the saturation pressure of water vapour is calculated accordingly to the Magnus-Teten-formula for water vapour over liquid water (Lowe 1977). That means:

$$
E_{\mathrm{w}}=A \exp \left(\frac{B(T-273.16 \mathrm{~K})}{T-C}\right)
$$

in which $A=610.78 \mathrm{~Pa}, B=17.2693882$ and $C=35.86 \mathrm{~K}$. Since liquid water does not currently exist on Mars the model has to be adjusted regarding this point. To account for this the saturation pressure is calculated with the Magnus-Teten-formula for water vapour over ice (Murray 1967), which is similar to Eq. (1) but with the values $A=610.66 \mathrm{~Pa}, B=21.875$ and $C=7.65 \mathrm{~K}$. When water condenses in the air it falls out instantaneously and is stored on the surface as snow under Mars conditions. Clouds are formed and affect radiation but do not store water. The cloud cover and cloud liquid water (equivalent) are derived as diagnostic variables from relative humidity. The scheme used for the cloud cover

$$
c c=\min \left[\left(\frac{r h-r h_{\mathrm{c}}}{1-r h_{\mathrm{c}}}\right)^{2}, 1\right],
$$


with relative humidity $r h$ and the critical relative humidity $r h_{\mathrm{c}}=0.85$ has been adapted from Slingo and Slingo (1991). Cloud liquid water equivalent $c l w \mathrm{in} \mathrm{kg} / \mathrm{kg}$ is derived in the following way:

$$
c l w=\frac{c l w^{0}}{\rho} \exp \left(-\frac{z}{h_{1}}\right)
$$

where $\rho$ is the density of air, $c l w^{0}=0.21 \times 10^{-3} \mathrm{~kg} / \mathrm{m}^{3}$ and $h_{1}$ the vertically integrated water vapour.

Local negative specific humidity which is an artifact of the spectral method, is corrected by a simple scheme which conserves the global amount of water by setting the specific humidity to zero at places where it is negative and by reducing the humidity globally by a factor equivalent to the water mass added. Evaporation from the surface is controlled by surface temperature, saturation pressure, wind speed and the properties of the surface:

$$
L F_{q}=L \rho C_{\mathrm{h}} C_{\mathrm{w}}|\vec{v}|\left(\delta q^{t+\Delta t}-q_{\mathrm{s}}\right),
$$

in which $L$ is the latent heat of sublimation, $F_{q}$ is the surface flux of moisture, $\rho$ is the density of air, $C_{\mathrm{h}}$ is the transfer coefficient of heat, $|\vec{v}|$ is the absolute value of horizontal velocity in the lower-most model layer, $\delta q^{t+\Delta t}$ the change of specific humidity in the lowest model layer, $q_{\mathrm{s}}$ is the saturation pressure at the surface and

$$
C_{\mathrm{w}}= \begin{cases}\min \left(\frac{W_{\text {soil }}}{f_{C \mathrm{w}} W_{\max }}, 1\right), & \text { soil, } \\ 1, & \text { ice or snow }\end{cases}
$$

is a "wetness"-factor accounting for the different evaporation efficiencies of the surface. In Eq. (5) $W_{\text {soil }}$ is the actual content of the ground and $W_{\max }=0.5 \mathrm{~m}$ is the maximum water capacity of the ground. The factor $f_{C \mathrm{w}}$ is set equal to 0.4 to facilitate a slow outgasing of water vapour from the regolith even though the water content of the regolith is minimal. $C_{\mathrm{w}}$ is very small for the regolith and is set to unity for the glacier covered north pole and snow covered ground. Furthermore, an assumption has to be made about the global water distribution in the regolith. For our experiments we use a globally uniform ground storage of $W_{\text {soil }}=0.0025 \mathrm{~m}$ liquid water equivalent at simulation start, which is not replenished, as water is accumulated on the ground as snow and no condensation of water vapour takes place underneath the surface. The heat transfer and drag coefficients are calculated based on Monin-Obukhov similarity theory following Louis (1979).

The amount of incoming solar radiation is based on the orbital configuration of the planet. Absorption, reflection and transmission of short wave radiation are calculated at each model layer in two distinctive bands, visible and near infra-red, respectively. 
Absorption due to dust in the atmosphere is not prescribed in the presented experiments to simulate a clear sky. Test runs with a globally uniform opacity larger than zero due to dust show a warmer and slightly moister atmosphere than in the experiments described below. Long wave radiation from the surface is partly absorbed in the atmosphere depending on water vapour distribution and by $\mathrm{CO}_{2}$ as a function of pressure. Clouds though only present as diagnostic conditions affect the radiation in the model due to reflection, absorption and emission depending on cloud cover and cloud liquid water equivalent as described above in Eqs. (2) and (3). At the present day atmospheric condition on Mars, water clouds with their typical optical thickness of $\tau=0.0$ to 0.5 , as described by Zasova et al. (2006), play only a small role in the global radiation budget (Zurek et al. 1992, page 864). This is not necessarily the case for past climate states of Mars. The models main purpose is to simulate long term climate changes and so radiative effects of clouds are included.

Planet Simulator Mars and SICOPOLIS are numerical models which operate on different spatial and time scales. This would make a direct coupling of the two models difficult and not very sensible because of the short term fluctuation of the atmosphere. The development of the ice cap is driven by the longterm climate mean. This legitimates the choice to force the ice model with climatological means from the atmospheric model.

\subsection{Interpolation of Planet Simulator Mars data onto the SICOPOLIS grid}

SICOPOLIS has a horizontal resolution of $91 \times 91$ grid points at a distance of $20 \mathrm{~km}$. The grid is centred on the pole. The Planet Simulator Mars uses a Gaußian grid of $64 \times 32$ points. Along a circle of latitude the distance between two gridpoints is $5.625^{\circ}$. On Mars at $80^{\circ}$ latitude this corresponds to a distance of $58 \mathrm{~km}$ and at $70^{\circ}$ to a distance of $114 \mathrm{~km}$. Along the meridians both the distance in degree and meter vary.

The grid resolution of SICOPOLIS is much higher than that of the Planet Simulator Mars. To prepare the boundary data from Planet Simulator Mars for the use with SICOPOLIS, an interpolation scheme has to be used. There are several ways to do this (e.g., Press et al. 1992). Herein a triangulation scheme as described by Cline and Renka (1984) is used.

\subsection{Model topography and boundary conditions}

In its normal configuration the Planet Simulator Mars uses an orography that has been derived from the MOLA topography (Segschneider et al. 2005, Smith et al. 1999). This 
includes, naturally, the ice caps. The Planet Simulator Mars uses a glacier map to distinguish between ice- and regolith-covered ground. In its basic configuration, this glacier map covers the northern most grid points of the model. The albedo depends on the surface characteristics. For regolith the albedo is uniformly set to 0.2 while for $\mathrm{CO}_{2}$ covered ground an albedo of 0.6 is being used. Snow covered regions obtain an albedo of 0.4 to 0.8 depending on depth. The heat capacity of the surface depends on the material, regolith, snow or ice, respectively. The heat capacity

$$
c_{\mathrm{top}}=\frac{c_{\text {snow }} c_{\text {soil }} z_{\text {top }}}{c_{\text {snow }} z_{\text {soil }}+c_{\text {soil }} z_{\text {snow }}}
$$

of the upper

$$
z_{\text {top }}=z_{\text {soil }}+z_{\text {snow }}=0.2 \mathrm{~m}
$$

is calculated from heat capacities of soil $\left(c_{\text {soil }}=1.07 \times 10^{6} \mathrm{~J} \mathrm{~m}^{-3} \mathrm{~K}^{-1}\right)$ and snow $\left(c_{\text {snow }}=\right.$ $\left.2.115 \times 10^{6} \mathrm{~J} \mathrm{~m}^{-3} \mathrm{~K}^{-1}\right)$ (Kossacki and Markiewicz 2002).

To set up different initial conditions for SICOPOLIS, e.g. a model start with no present north polar ice cap, the same has to be done for the Planet Simulator Mars too. So the glacier map and the orography have to be adjusted accordingly.

Since the bedrock topography under the ice cap is unknown, it is interpolated from the surrounding topography (Greve et al. 2004, Mahajan 2005). A simple solution for an experiment without initial ice cap is to interpolate the bed rock topography for the Planet Simulator Mars as well. Figure 1 depicts the Planet Simulator Mars orography with and without the present ice cap.

(a)

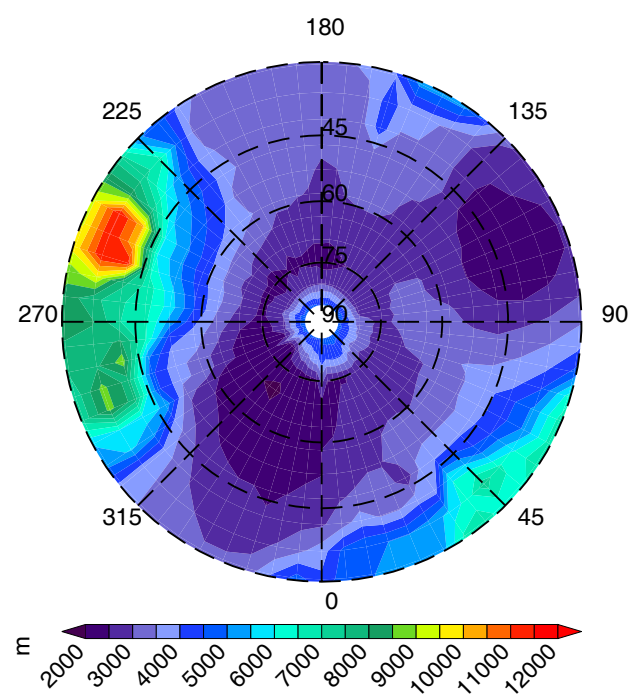

(b)

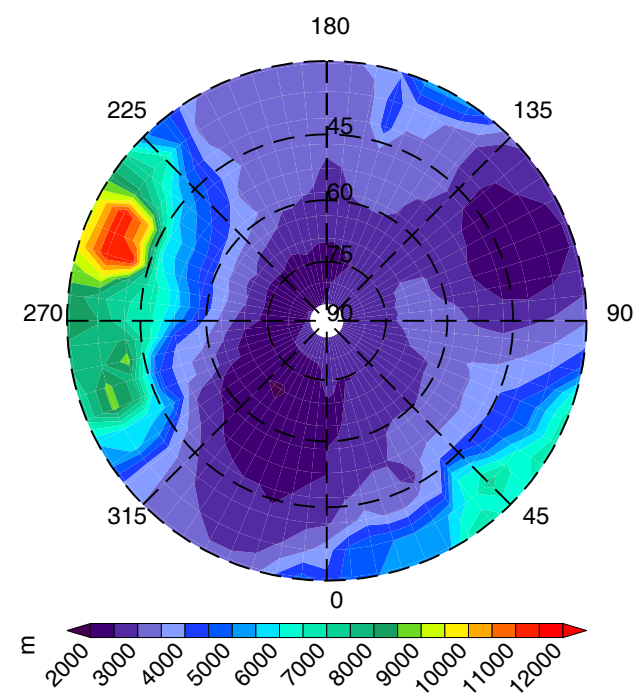

Figure 1: The Planet Simulator Mars orography (a) with and (b) without the north polar ice cap. The elevations give the heights above the lowest point in the model domain rather than the deviation from the reference geoid. 


\section{Experiments}

Two series of experiments are carried out with this coupled atmosphere-icesheet model to probe the impact of secular climate changes on the north polar ice-cap. In the first experiment (Sec. 3.1) we assess the impact of different obliquities on the present north polar cap. The second set of experiments (Sec. 3.2) looks into the question under which obliquity constraints a new north polar ice-cap can be formed.

Our experiments are carried out with three constant obliquity angles and not with slowly varying inclination of the rotation axis to save computation time and to understand the steady-state climate condition at a given obliquity. For the future longer experiments with transient changes of obliquity, eccentricity, and longitude of vernal equinox are planed. We chose obliquities of $15^{\circ}, 25^{\circ}$, and $35^{\circ}$, because they represent the minimum, average (and present day) and maximum obliquities in the last 4 million years (Laskar et al. 2004).

\subsection{Experiment (ICE) with present ice cap}

These experiments simulate the development of the north polar cap (NPC) over the time-span of 25 million years, the atmospheric fields being computed by the Planet Simulator Mars by using the present day topography shown in Fig. 1a. Note that, by contrast, SICOPOLIS starts from an ice free ground. The coupling in this experiment is working in one direction only: The atmospheric model's average fields are used as a constant boundary condition for the ice sheet model.

In the first step, as detailed in section 2, the atmospheric part of the model is integrated with three different constant obliquity values for two years:

1. $\mathbf{I C E}_{15}$ with $\theta=15^{\circ}$,

2. $\mathbf{I C E}_{\mathbf{2 5}}$ with $\theta=25^{\circ}$, and

3. $\mathbf{I C E}_{\mathbf{3 5}}$ with $\theta=35^{\circ}$.

All other boundary conditions are identical in the three model runs. The eccentricity is fixed at $e=0.093$ and the longitude of perihelion relative to vernal equinox is set to $v_{\mathrm{e}}=250^{\circ}$. From these runs the second years are used in a second step to calculate the annual mean temperature (Fig. 2) and mass-balance (Fig. 3) fields to be used in the subsequent ice-model integrations. In this set-up, three experiments are conducted with SICOPOLIS using the three datasets from Planet Simulator Mars. As the forcing fields are constant through out the integration with SICOPOLIS the amount of water on Mars is not conserved. 

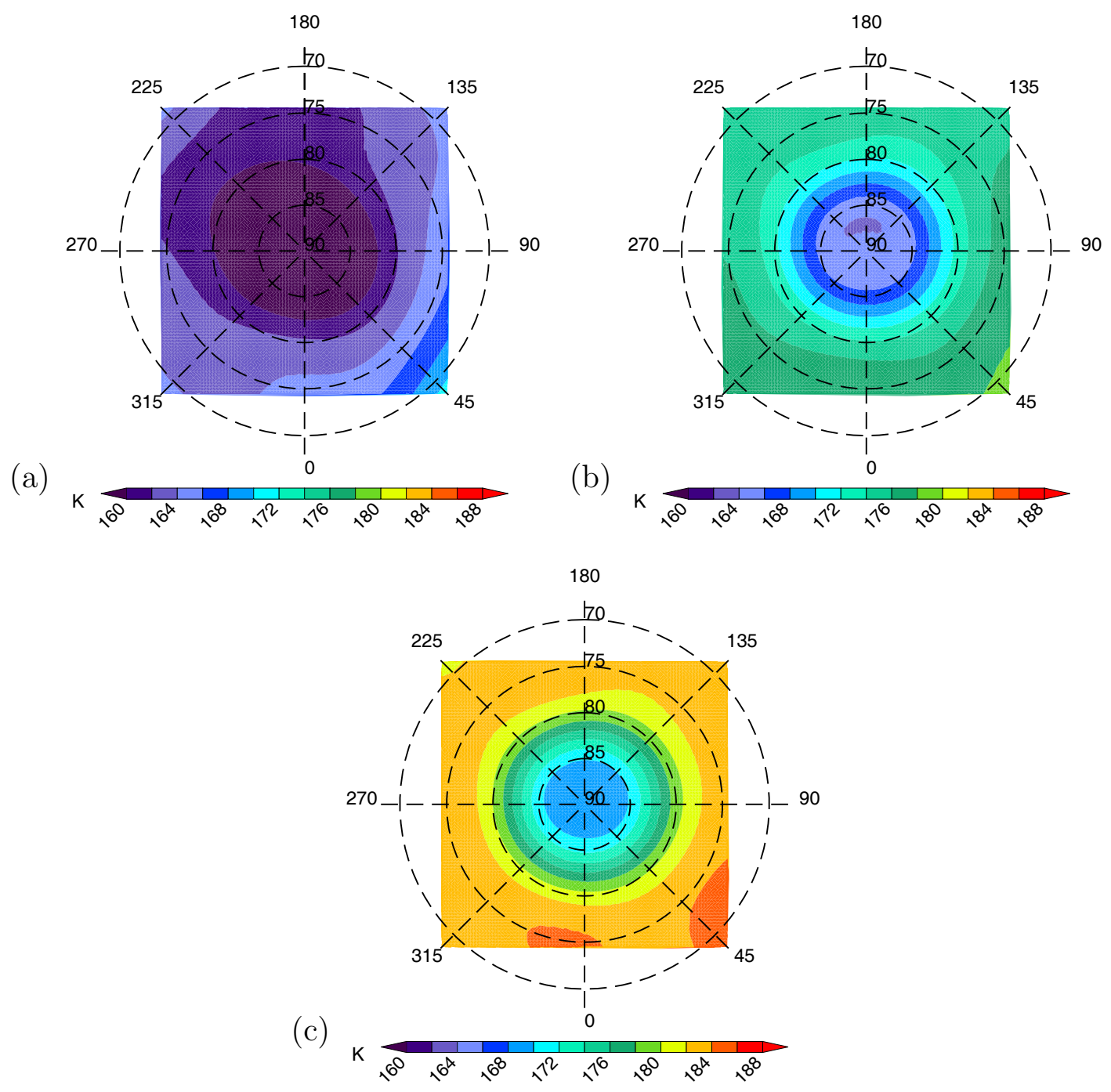

Figure 2: Annual means of temperature $2 \mathrm{~m}$ above the ground from the three ICE experiments at (a) $\theta=15^{\circ}$, (b) $25^{\circ}$ and (c) $35^{\circ}$, respectively. The actual results from the Planet Simulator Mars are interpolated onto the SICOPOLIS grid centred at the north pole. 

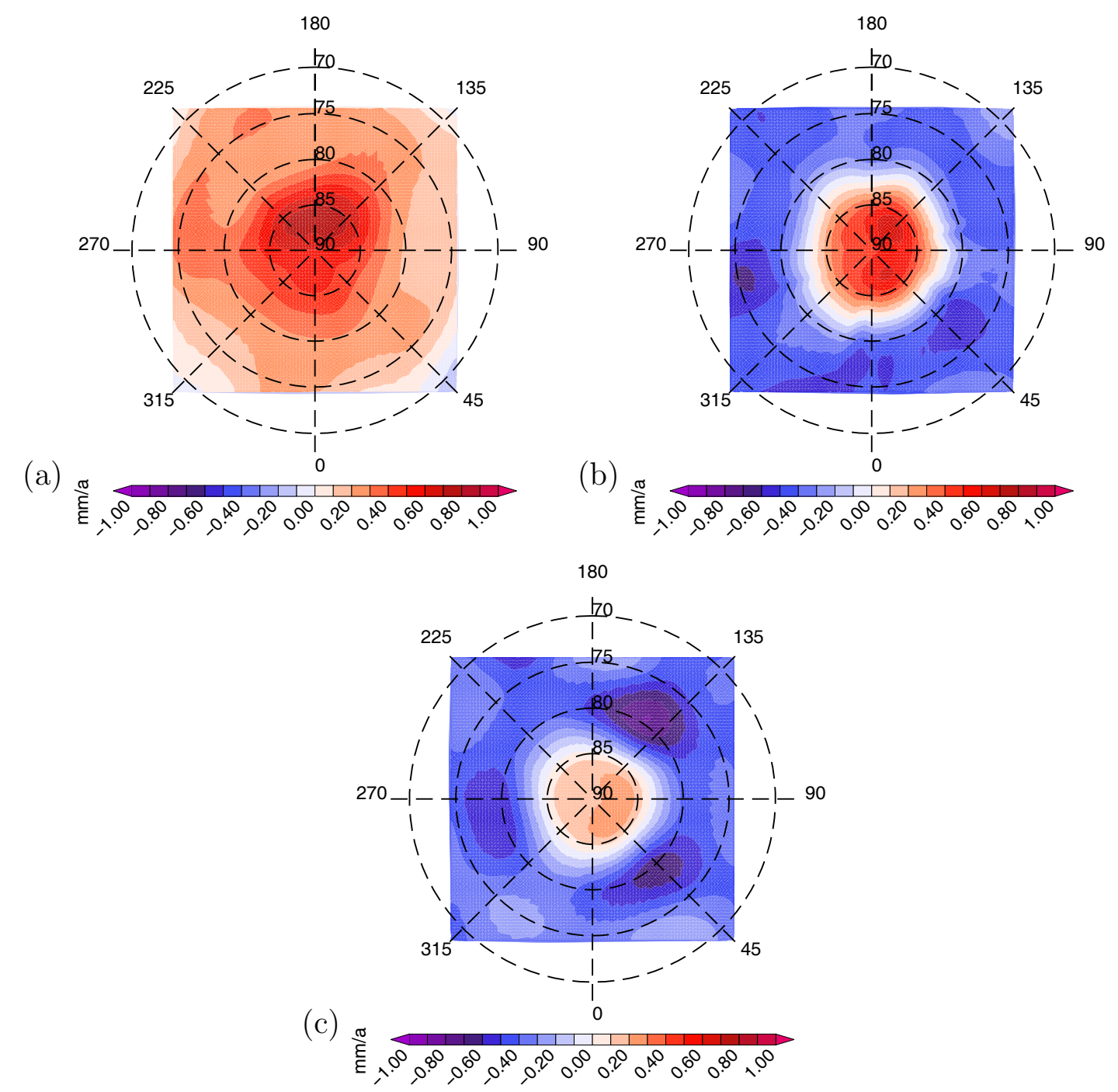

Figure 3: Mass-balance from the Planet Simulator Mars for the three ICE experiments, interpolated onto the SICOPOLIS grid centred at the north pole at (a) $\theta=15^{\circ}$, (b) $25^{\circ}$ and (c) $35^{\circ}$, respectively. 


\subsection{Experiment (NO-ICE) with no initial ice cap}

To test the impact of the presence or absence of the NPC on the atmospheric circulation at different obliquities, we present now an experiment with the coupled Planet Simulator Mars, that starts with no initial NPC. Or to put it in another way: We test how the coupling in the SICOPOLIS to Planet Simulator Mars direction works, as the prescribed glacier and albedo maps are normally obtained from the ice sheet model, although in this experiment the glacier and albedo maps are given as a starting condition and are not results from a previous SICOPOLIS run. Another question we try to answer with this experiment is, whether or at what obliquity a new NPC can be built after a complete ablation during periods of very large obliquity.

A similar scheme as the one used to interpolate the Planet Simulator Mars data onto the SICOPOLIS grid is now used to estimate the orography underneath the present ice cap. The glacier mask is set to zero which implies that the albedo map is changed in the north polar region to yield the regolith value. These are the only changes made from the ICE experiment. Planet Simulator Mars is integrated with these boundary data with constant obliquities of $\theta=25^{\circ}, 15^{\circ}$, and $35^{\circ}$ each for two model years:

1. NO-ICE $\mathbf{1 5}_{15}$ with $\theta=15^{\circ}$,

2. $\mathbf{N O}-\mathbf{I C E}_{\mathbf{2 5}}$ with $\theta=25^{\circ}$, and

3. NO-ICE $\mathbf{H 5}_{35}$ with $\theta=35^{\circ}$.

Of the two years only the second one is used for analysis and the subsequent SICOPOLIS runs. The ice-model is again integrated for each of the three obliquities under constant boundary conditions for 25 million years. Like in the ICE experiment the forcing fields are constant through out the integration with SICOPOLIS, so the amount of water on Mars is not conserved.

\section{Results and discussion}

Looking at the results from the three ICE experiments, a strong dependence of near surface air temperatures on obliquity can be found at all latitudes (Fig. 4a). While the polar regions get warmer the lower latitudes are becoming colder as the obliquity increases. The water content of the atmosphere is less affected by obliquity in the tropics than in the polar regions. The $\mathbf{I C E}_{\mathbf{2 5}}$ experiment shows the highest humidity (Fig. 4b). This is a bit surprising when compared to the experiments by Mischna et al. (2003), who find a vastly higher atmospheric water vapour content for $\theta=35^{\circ}$. The reasons stem 
(a)

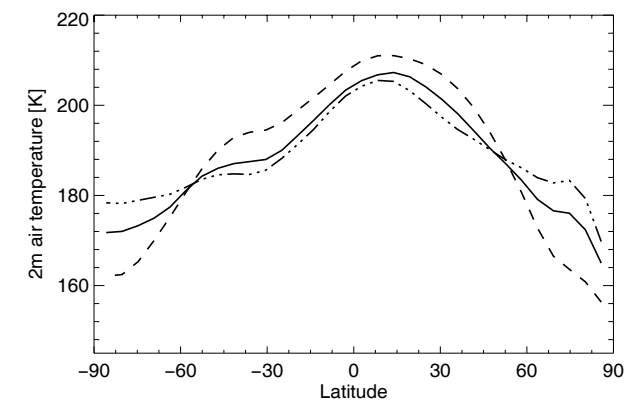

(b)

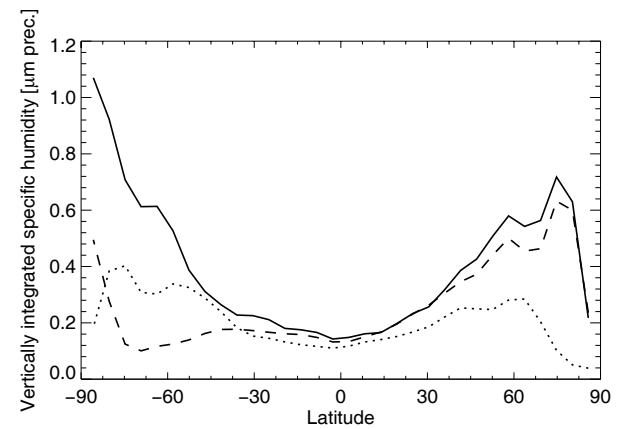

Figure 4: (a) Zonal mean surface air temperature and (b) vertically integrated specific humidity for the three ICE experiments. All graphs show annual mean values. The dashed, solid and dash-dotted lines represent the obliquities of $\theta=15^{\circ}, 25^{\circ}$ and $35^{\circ}$, respectively.

(a)

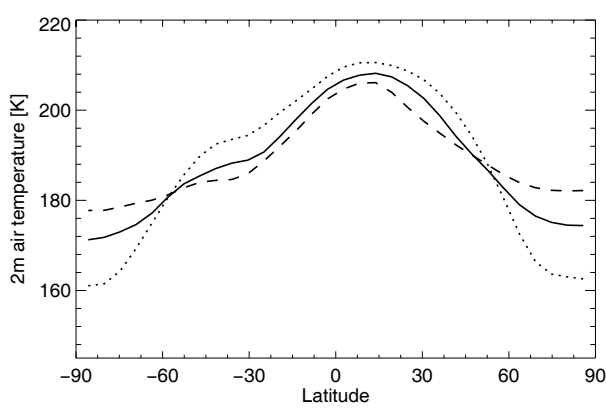

(b)

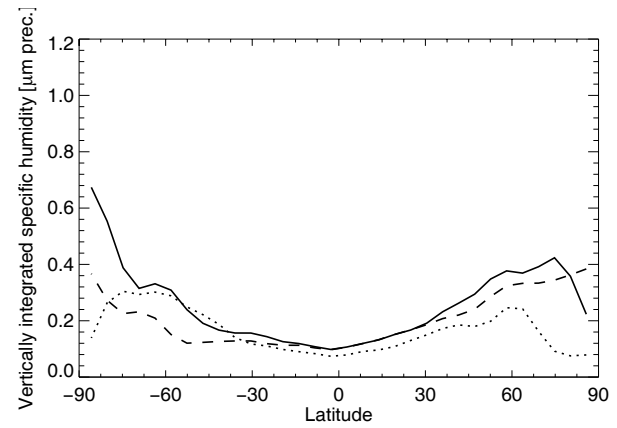

Figure 5: (a) Zonal mean surface air temperature and (b) vertically integrated specific humidity for the three NO-ICE experiments. All graphs show annual mean values. The dashed, solid and dash-dotted lines represent the obliquities of $\theta=15^{\circ}, 25^{\circ}$ and $35^{\circ}$, respectively.

from different boundary conditions for the simulation of the hydrological cycle. Mischna et al. are generally using the parametrisations described in the work of Richardson and Wilson (2002a); this includes a NPC covering the two northern most latitudes at $87.5^{\circ} \mathrm{N}$ and $82.5^{\circ} \mathrm{N}$ of the GFDL Mars-model. Planet Simulator Mars' north polar ice cap is smaller, consisting of the grid points at $85.761^{\circ} \mathrm{N}$. The different extension of the ice cap results in different evaporation rates. Further, Mischna et al. are using a water ice albedo of $\alpha=0.38$, which is the minimum albedo obtained in summer by James and Cantor (2001) while the present study uses water ice albedo of $\alpha=0.8$, which corresponds to the albedo of glacier surface on Earth. A further difference between the two models is the treatment of latent heat. Following Zurek et al. (1992), Richardson and Wilson (2002a) and Mischna et al. (2003) make the assumption that the heat from evaporation or sublimation plays only an insignificant role in Martian climate system as the amounts of water involved are very small. For this reason, their model neglects latent heat. Planet Simulator Mars, on the other hand, simulates the heating or cooling due to sublimation and evaporation, resulting in a negative feedback for atmospheric 
water vapour content. In the cases of low and medium obliquities with the associated low insolation in the polar regions, the heat fluxes involved with the phase change of water are small but for higher obliquities with higher insolation involved, the assumption is not necessarily valid.

This unidirectionally coupled run gives a good first impression on the capability of the fully coupled model. Different from the SICOPOLIS experiments without Planet Simulator Mars (Greve et al. 2004), the SICOPOLIS runs from ICE show an ice cap that is not symmetric around the pole illustrating the impact of atmospheric circulation on the shape of the ice cap. This can be deduced from the different forcings used in the two studies. While Greve et al. (2004) uses topographic data as in the present work, the atmospheric forcings which are surface temperature, accumulation and ablation depend purely on radiation balance. In case the mass balance would be controlled only by the radiation budget, the ablation and accumulation would be purely functions of latitude but with atmospheric circulation included, water vapour is transported from the place of its origin and condenses elsewhere. Condensation takes place where the air is ascending either forced by topography as can been seen at the large volcanos or by convergence of the flow in the lower altitudes which are part of the meridional circulation. Of great importance for the high precipitation rates over the north pole in our simulation is a cross polar flow that branches out of the circum polar planetary wave. Longitudinal temperature variations at the surface play only a minor role.

The impact of different obliquity angles can clearly be seen: The three experiments give different mean growth rates in the first five million years of about $0.85 \mathrm{~mm} / \mathrm{a}$, $0.74 \mathrm{~mm} / \mathrm{a}$ and $0.33 \mathrm{~mm} / \mathrm{a}$ for $\mathbf{I C E}_{\mathbf{1 5}}, \mathbf{I} \mathbf{C E}_{\mathbf{2 5}}$, and $\mathbf{I} \mathbf{C E}_{\mathbf{3 5}}$, respectively.

At $\theta=15^{\circ}$ the model results show the highest and farthest extending ice cap from the three integrations. It reaches down to nearly $70^{\circ} \mathrm{N}$ and grows during the simulation period of 5 million years to a maximum thickness of about $4.3 \mathrm{~km}$ (Fig. 6a). For $\theta=25^{\circ}$ the ice cap extends southward to $83^{\circ} \mathrm{N}$ and so is a bit smaller than the actual one. After 5 million years it reaches a maximum thickness of $3.7 \mathrm{~km}$ (Fig. 6b). At $\theta=35^{\circ}$ SICOPOLIS produces a very small ice cap that covers the pole down to $85^{\circ} \mathrm{N}$ and has a maximum thickness of just about $1.6 \mathrm{~km}$ (Fig. 6c).

The NO-ICE experiment results in a quite similar meridional mean temperature distribution outside the north polar region where ICE exhibits lower near surface temperatures due to the higher albedo (Fig 5a). While the humidity in the lower latitudes is approximately the same in both scenarios, the higher latitudes show much less atmospheric water content (Fig 5b). As no perennial water ice cap is present in NO-ICE, the water vapour in the atmosphere is replenished only by sublimation from the regolith, depending on temperature of the surface and stability of the lower-most atmospheric 
(a)

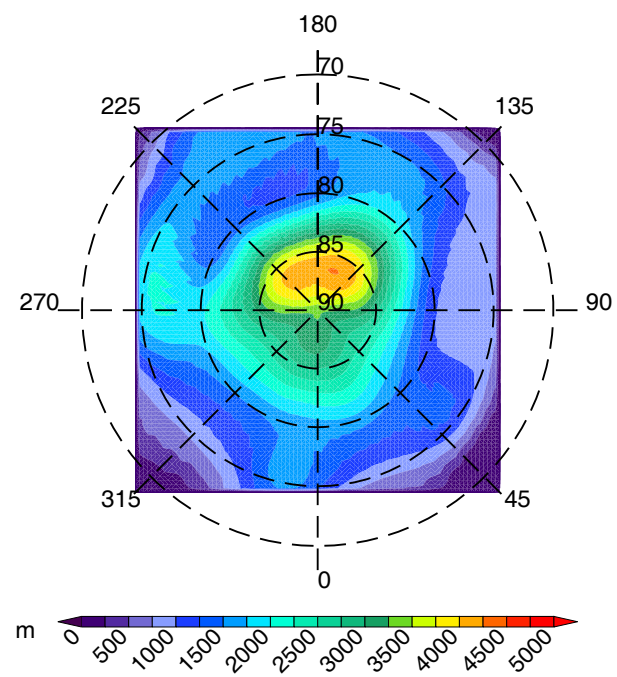

(b)

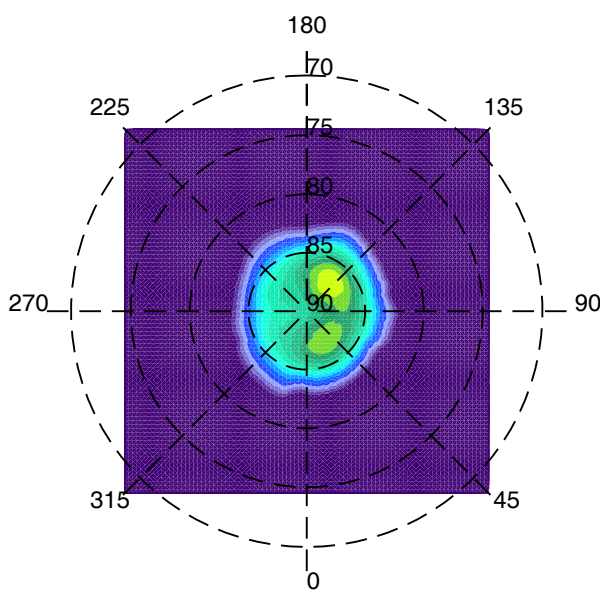

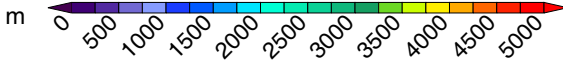

(c)

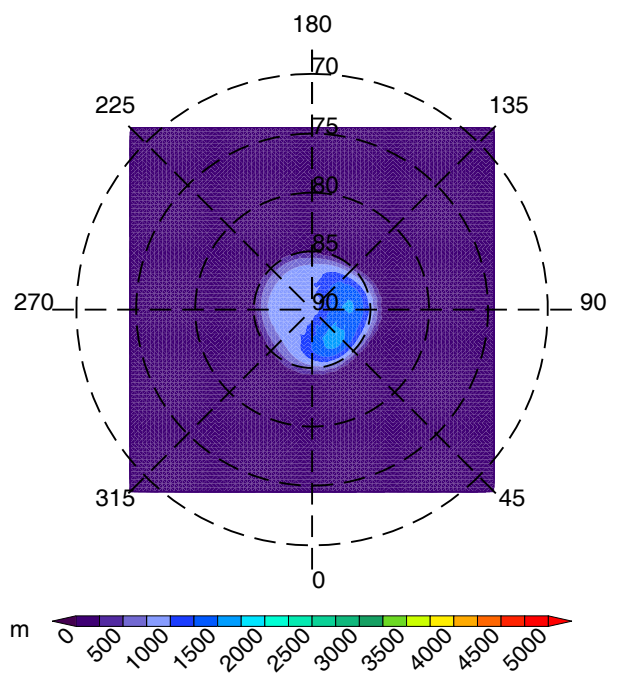

Figure 6: Thickness of the ice cap after $5 \mathrm{Ma}$ for the three ICE experiments at (a) $\theta=15^{\circ}$, (b) $25^{\circ}$ and (c) $35^{\circ}$, respectively.

layer.

Figure 7 shows the precipitation - evaporation (P-E) for NO-ICE $\mathbf{2 5}_{\mathbf{2 5}}$. The temperatures around the north pole are significantly higher than in $\operatorname{run}_{\mathbf{I}} \mathbf{C E}_{\mathbf{2 5}}$ from the $\mathbf{I C E}$ experiment (Fig. 2b, page 9). The mass balance for the ground (P-E) is negative for almost the whole polar region. That implies that at an obliquity of $\theta=25^{\circ}$ no new perennial ice cap can be build. The comparison with ICE shows on the other end the growth of an already existing polar cap at the same obliquity. At $\theta=35^{\circ}$ we observe the same condition: the growth of a new ice cap is not possible.

Further experiments with lower inclinations of the rotation axis can show at what point it is possible to build up a new polar cap. At an obliquity of $\theta=15^{\circ}$ the models show a new cap. So the point of 'critical' obliquity $\theta_{\text {crit }}$ lies between $15^{\circ}$ and $25^{\circ}$. To find 


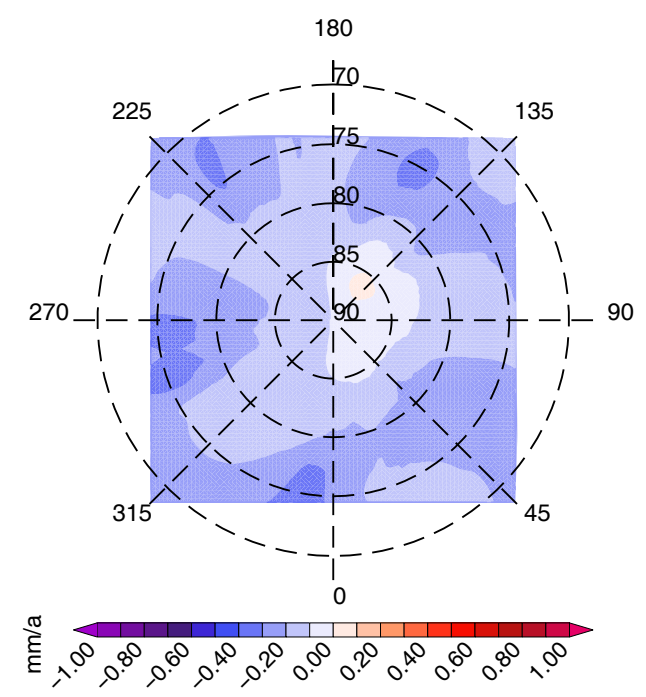

Figure 7: Mass-balance from the Planet Simulator Mars in the NO-ICE experiment, run NO-ICE $\mathbf{I}_{25}$.

this $\theta_{\text {crit }}$, we examine the obliquity range between $\theta=15^{\circ}$ and $\theta=25^{\circ}$. We are using nested-intervals to check for $\theta_{\text {crit }}$. In this way we can confine the 'critical' obliquity to $\theta_{\text {crit }}=22^{\circ}$. To estimate at which obliquity an existing ice vanishes, experiments with obliquities larger than $\theta=35^{\circ}$ have also been conducted up to obliquities of $\theta=50^{\circ}$. They show no distinct upper boundary for the existence of a north polar ice cap.

The presented experiments examine long term constant climate conditions at fixed orbital parameters which do not exist in the real world. A more realistic approach would be to slightly change orbital parameters between model runs and allow for a bidirectional feedback between the atmospheric and the ice part, as it is planned for the future. How the results would differ is not easy to forecast, but the following results could be expected to be probable: The albedo feedback is likely to accelerate the growth and the waning of the ice cap. The threshold obliquity that triggers the build of a new ice cap would not necessarily be the same obliquity found in this work but can be expected to exist.

\section{Conclusions}

From our model results it seems that the north polar cap (NPC) is sensitive to changes in the obliquity of the planet. For the present cap at current orbital condition a growth of the cap in the centre and a negative mass balance at the fringes are predicted. At lower obliquity $\left(15^{\circ}\right)$ the NPC spreads further southward and at higher obliquity $\left(35^{\circ}\right)$ it is only present in a small area around the pole. The ice lost from the poles is deposited in the lower latitudes. These results are in good comparison to what has been expected based on satellite observations (Head et al. 2003). One can speculate that only an 
ice cap that does not vanish during high obliquity periods, which can reach $\theta=60^{\circ}$ (Laskar et al. 2004), can grow through tens of millions of years and reach its current thickness. The low accumulation rates are a result of the low water content of the models simulated atmosphere. The atmospheric temperatures in the polar regions of Planet Simulator Mars are lower than in the real Martian atmosphere. This is due to the clear atmosphere approach we are taking here and the missing variation of atmospheric $\mathrm{CO}_{2}$ content. Low temperatures again result in low water content. The approach of a constant global mean surface pressure can lead to unrealistic temperature distributions and meridional circulation patterns, especially in the polar night atmosphere. However the simple parametrisation of a cut off temperature at $148 \mathrm{~K}$ which controls the seasonal frost caps mitigates some of the problems created by using a constant $\mathrm{CO}_{2}$ atmosphere. Comparing with Mars Global Surveyer Thermal Emission Spectrometer (TES) results (Smith 2004, 2006), Planet Simulator Mars underestimates atmospheric water by a factor of 5 to 10. However, the distribution of water vapour is well captured in our model apart from the high southern sub polar water content which is largely an artifact of the model spin-up and is reduced in subsequent years for longer integration than the cases presented here (Fig. 4). For the experiments presented here, which deal with the water budget in the higher latitudes of the northern hemisphere, the impact of the different water vapour contents in the southern hemisphere is small, so we think that our results are qualitatively reliable. As the model is further improved the problems with the water content should vanish.

So far a functional coupled model of the Martian atmospheric general circulation has been developed within this project. The atmospheric model has been coupled to the ice sheet model SICOPOLIS. The Planet Simulator Mars now simulates successfully a humid Martian atmosphere with a low dust content, the change of surface albedo due to seasonal $\mathrm{CO}_{2}$ caps and perennial water ice caps in dependence of the orbital parameters of Mars. However the development of Planet Simulator Mars / SICOPOLIS is being continued. At the time this paper is written, the effects of dust as an absorber in the atmosphere is included and a simple but more realistic $\mathrm{CO}_{2}$ cycle has been implemented. In SICOPOLIS the model domain has been enlarged to cover glaciations in the higher mid-latitudes. For the future we aim to use this improved version to simulate climate cycles due to secular orbital changes.

\section{Acknowledgments}

We would like to thank two anonymous reviewers for their valuable comments and criticisms. This work has been funded by the DFG within its priority programme "Mars 
and the terrestrial planets", and by the Institute of Low Temperature Science (Sapporo, Japan) within the project "Evolution and dynamics of the Martian polar ice caps over climatic cycles".

\section{References}

Armstrong, J. C., C. B. Leovy and T. Quinn. 2004. A 1 Gyr climate model for Mars: new orbital statistics and the importance of seasonally resolved polar processes. Icarus, 171, 255-271.

Cline, A. K. and R. L. Renka. 1984. A storage-efficient method for construction of a Thiessen triangulation. Rocky Mountain Journal of Mathematics, 14 (1), 119-139.

Forget, F., F. Hourdin, R. Fournier, C. Hourdin, O. Talagrand, M. Collins, S. R. Lewis, P. L. Read and J.-P. Huot. 1999. Improved general circulation models of the Martian atmosphere from the surface to above $80 \mathrm{~km}$. Journal of Geophysical Research, 104 (E10), 24155-24175.

Fraedrich, K., H. Jansen, E. Kirk, U. Luksch and F. Lunkeit. 2005a. The Planet Simulator: Towards a user friendly model. Meteorologische Zeitschrift, 14 (3), 299-304.

Fraedrich, K., H. Jansen, E. Kirk and F. Lunkeit. 2005b. The Planet Simulator: Green planet and desert world. Meteorologische Zeitschrift, 14 (3), 305-314.

Gierasch, P. and R. Goody. 1968. A study of the thermal and dynamical structure of the martian lower atmosphere. Planetary and Space Sciences, 16, 615-646.

Greve, R. 1997. Application of a polythermal three-dimensional ice sheet model to the Greenland ice sheet: Response to steady-state and transient climate scenarios. Journal of Climate, 10 (5), 901-918.

Greve, R. 2006. Fluid dynamics of planetary ices. GAMM-Mitteilungen, 29 (1), 29-51.

Greve, R. and R. Mahajan. 2005. Influence of ice rheology and dust content on the dynamics of the north-polar cap of Mars. Icarus, 174 (2), 475-485.

Greve, R., R. A. Mahajan, J. Segschneider and B. Grieger. 2004. Evolution of the northpolar cap of Mars: a modelling study. Planetary and Space Science, 52, 775-787.

Head, J. W., J. F. Mustard, M. A. Kreslavsky, R. E. Milliken and D. R. Marchant. 2003. Recent ice ages on Mars. Nature, 426, 797 - 802. 
Hutter, K. 1983. Theoretical Glaciology; Material Science of Ice and the Mechanics of Glaciers and Ice Sheets. D. Reidel Publishing Company, Dordrecht, The Netherlands.

Jakosky, B. M. 1985. The seasonal cycle of water on Mars. Space Science Reviews, 41, $131-200$.

James, P. B. and B. A. Cantor. 2001. Martian north polar cap recession: 2000 Mars Orbiter Camera observations. Icarus, 154, 131-144.

Kossacki, K. J. and W. J. Markiewicz. 2002. Martian seasonal $\mathrm{CO}_{2}$ ice in polygonal troughs in southern polar region: Role of the distribution of subsurface $\mathrm{H}_{2} \mathrm{O}$ ice. Icarus, 160 (1), 73-85.

Laskar, J., A. C. M. Correiaa, M. Gastineaua, F. Joutela, B. Levrarda and P. Robutela. 2004. Long term evolution and chaotic diffusion of the insolation quantities of Mars. Icarus, 170 (2), 343 - 364.

Laskar, J., B. Levrad and J. F. Mustard. 2002. Orbital forcing of the martian polar layered deposits. Nature, 419, 375-377.

Louis, J. F. 1979. A parametric model of vertical eddy fluxes in the atmosphere. Boundary Layer Meteorology, 17, 187-202.

Lowe, P. R. 1977. An aproximating polynomial for the computation of saturation vapor pressure. Journal of Applied Meteorology, 16, 100-103.

Lunkeit, F., M. Böttinger, K. Cassirer, K. Fraedrich, H. Jansen, W. Joppich, E. Kirk, A. Kleidon, M. Lob, U. Luksch and M. Preuhs. 2004. Planet Simulator Reference Manual. Universität Hamburg, Bundesstr. 55, D-20146 Hamburg.

Mahajan, R. A. 2005. Modelling the Martian Polar Caps. Ph.D. thesis, Universität Göttingen

Michelangeli, D. V., O. B. Toon, R. M. Haberle and J. B. Pollack. 1993. Numerical simulations of the formation and evolution of water ice clouds in the Martian atmosphere. Icarus, 102 (2), 261-285.

Mischna, M. A., M. I. Richardson, R. J. Wilson and D. J. McCleese. 2003. On the orbital forcing of Martian water and $\mathrm{CO}_{2}$ cycles: A general circulation model study with simplified volatile schemes. Journal of Geophysical Research - Planets, 108 (E6). DOI 10.1029/2003JE002051. 
Morland, L. W. 1984. Thermo-mechanical balances of ice sheet flows. Geophysical and Astrophysical Fluid Dynamics, 29, 237-266.

Murray, F. W. 1967. On the computation of saturation vapor pressure. Journal of Applied Meteorology, 6, 203-204.

Petoukhov, V., A. Ganopolski, V. Brovkin, M. Claussen, A. Eliseev, C. Kubatzki and S. Rahmstorf. 2000. Climber-2: a climate system model of intermediate complexity. Part I: model description and performance for present climate. Climate Dynamics, $16(1), 1-17$.

Pollack, J. B., M. Haberle, J. Schaeffer and H. Lee. 1990. Simulation of the general circulation of the Martian atmosphere. I. Polar processes. Journal of Geophysical Research, 95, 1447-1473.

Press, W. H., S. A. Teukolsky, W. T. Vetterling and B. P. Flannery. 1992. Numerical Recipies in Fortran - The Art of Scientific Computing. Cambridge University Press, $2^{\text {nd }}$ ed.

Read, P. L., M. Collins, F. Forget, R. Fournier, F. Hourdin, S. R. Lewis, O. Talagrand, F. W. Taylor and N. P. J. Thomas. 1997. A GCM climate database for Mars: for mission planning and for scientific studies. Advances in Space Research, 19, 12131222 .

Richardson, M. I. and R. J. Wilson. 2002a. Investigation of the nature and stability of the Martian seasonal water cycle with a general circulation model. Journal of Geophysical Research - Planets, 108 (E6). DOI 10.1029/2001JE001536.

Richardson, M. I. and R. J. Wilson. 2002b. A topographically forced asymmetry in the Martian circulation and climate. Nature, 416, 298-301.

Segschneider, J., B. Grieger, H. U. Keller, F. Lunkeit, E. Kirk, K. Fraedrich, A. Rodin and R. Greve. 2005. Response of the intermediate complexity Mars Climate Simulator to different obliquity angles. Planetary and Space Sciences, 53 (6), 659-670.

Slingo, A. and J. M. Slingo. 1991. Response of the National Center for Atmospheric Research community climate model to improvements in the representation of clouds. Journal of Geophysical Research, 96, 341 - 357.

Smith, D. E., M. T. Zuber, S. C. Solomon, R. J. Phillips, J. W. Head, J. B. Garvin, W. B. Banerdt, D. O. Muhleman, G. H. Pettengill, G. A. Neumann, F. G. Lemoine, J. B. Abshire, O. Aharonson, C. D. Brown, S. A. Hauck, A. B. Ivanov, P. J. McGovern, H. J. 
Zwally and T. C. Duxbury. 1999. The global topography of Mars and implications for surface evolution. Science, 284, 1495-1503.

Smith, M. D. 2004. Interannual variability in TES atmospheric observations of Mars during 1999-2003. Icarus, 167 (1), 148-165.

Smith, M. D. 2006. TES atmospheric temperature, aerosol optical depth, and water vapor observations 1999-2004. In: Second International Workshop on Mars Atmosphere Modeling and Observations. URL http://wwwmars.lmd.jussieu.fr/granada2006/abstracts/Smith2_Granada2006.pdf.

Stenzel, O. J. and J.-S. von Storch. 2004. On the effect of thermal forcing on the global atmospheric angular momentum and the general circulation. Climate Dynamics, 22 (4), 415-427.

Zasova, L. V., V. Formisano, V. I. Moroz, J.-P. Bibring, D. Grassi, N. Ignatiev, M. Giuranna, G. Bellucci, F. Altieri, M. Blecka, V. N. Gnedykh, A. V. Grigoriev, E. Lellouch, A. Mattana, A. Maturilli, B. E. Moshkin, Y. V. Nikolsky, D. V. Patsaev, G. Piccioni, M. Ratai, B. Saggin, S. Fonti, I. V. Khatuntsev, H. Hirsh and A. P. Ekonomov. 2006. Results of measurements with the planetary fourier spectrometer onboard Mars Express: Clouds and dust at the end of southern summer. A comparison with OMEGA images. Cosmic Research, 44 (4), 319-331.

Zurek, R. W., J. R. Barnes, R. M. Haberle, J. B. Pollack, J. E. Tillman and C. B. Leovy. 1992. Mars, chap. 26. Dynamics of the atmosphere of Mars, pp. 835-933. The University of Arizona Press, Tucson. 